\title{
Cdk5 Phosphorylation of STAT3 in Dorsal Root Ganglion Neurons Is Involved in Promoting Axonal Regeneration After Peripheral Nerve Injury
}

\author{
Jinyeon Hwang, Uk Namgung \\ Department of Korean Medicine, Daejeon University, Daejeon, Korea
}

Purpose: The goal of this study is to investigate the role of cyclin-dependent kinase 5 (Cdk5) in axonal regeneration in dorsal root ganglion (DRG) neurons after peripheral nerve injury.

Methods: Crush injury was given on the sciatic nerve in rats. The DRG tissues were prepared 1,3, and 7 days after injury and used for western blotting and immunofluorescence staining experiments. Primary DRG neurons were prepared and treated with Cdk5 inhibitor roscovitine or used for transfections with plasmid constructs. After immunofluorescence staining, neurite length of DRG neurons was analyzed and compared among experimental groups. In addition, roscovitine was injected into the DRG in vivo, and the sciatic nerve after injury was prepared and used for immunofluorescence staining to analyze axonal regeneration in nerve sections.

Results: Levels of Cdk5 and p25 were increased in DRG neurons after sciatic nerve injury (SNI). Levels of S727-p-STAT3, but not Y705-p-STAT3, were increased in the DRG. Immunofluorescence staining revealed that Cdk5 and STAT3 proteins were mostly colocalized in DRG neurons and Y705-p-STAT3 signals were localized within the nucleus area of DRG neurons. A blockade of Cdk 5 activity by roscovitine or by transfection with dominant negative Cdk5 (dn-Cdk5) and nonphosphorylatable forms of STAT3 (S727A or Y705F) resulted in significant reductions of the neurite outgrowth of cultured DRG neurons. In vivo administration of roscovitine into the DRG markedly attenuated distal elongation of regenerating axons in the sciatic nerve after injury.

Conclusions: Our study demonstrated that Cdk5 activity induced from DRG neurons after SNI increased phosphorylation of STAT3. The activation of Cdk5-STAT3 pathway may be involved in promoting axonal regeneration in the peripheral nerve after injury.

Keywords: Axonal regeneration; Cyclin-deponent kinase 5; Dorsal root ganglion; Sciatic nerve; STAT3

- Fund/Grant Support: This work was supported by the Daejeon University fund (grant number 20190123) (Daejeon, Korea).

- Research Ethics: All protocols involving the care of live animals and their postoperative care were approved by the Daejeon University Institutional Animal Use and Care Committee and were in accordance with the Animal-Use Statement and Ethics Committee Approval Statement for Animal Experiments provided by Daejeon University.

- Conflict of Interest: No potential conflict of interest relevant to this article was reported.

\section{- HIGHLIGHTS \\ - Cdk5 phosphorylates STAT3 protein in DRG sensory neurons after sciatic nerve injury in rats. \\ - Cdk5-STAT3 signal pathway is involved in facilitating axonal regeneration.}

Corresponding author: Uk Namgung (D) https://orcid.org/0000-0001-5253-1143 Department of Oriental Medicine, Daejeon University, 62 Daehak-ro, Dong-gu, Daejeon 34520, Korea

E-mail: unamgung@dju.kr / Tel: +82-42-280-2614 / Fax: +82-42-274-2600

Submitted: April 2, 2020 / Accepted after revision: May 7, 2020
This is an Open Access article distributed under the terms of the Creative Commons Attribution Non-Commercial License (http://creativecommons.org/licenses/by-nc/4.0/) which permits unrestricted non-commercial use, distribution, and reproduction in any medium, provided the original work is properly cited. 


\section{INTRODUCTION}

Regenerative responses in axons after peripheral nerve injury (PNI) are distinct from those in the central nervous system in which the injured axons have a limited capacity of regeneration thus failing to make a correct targeting [1]. Comparative analysis on axonal regeneration has revealed that both intrinsic neuronal and environmental factors respond differently to the injury between peripheral and central nervous systems [2-4]. For instance, Schwann cells and molecular factors produced by Schwann cells contribute to axonal regeneration $[5,6]$, whereas molecules such as Nogo, MAG, and OMgp that are produced from oligodendrocytes after spinal cord injury inhibit axonal regeneration [7].

Sensory neurons in the DRG have been widely used to investigate intrinsic molecular factors that are important for axonal regeneration. Neurotrophic factor signals such as nerve growth factor, glial cell-derived neurotrophic factor, and brain-derived neurotrophic factor are produced from Schwann cells and injured axons after PNI, entrapped with receptors, and retrogradely transported into the neuronal cell body to induce gene expression $[3,8,9]$. Axonal growth-associated protein 43 (GAP43) is one of the prototypical proteins which is induced at gene expression level in neurons after PNI [10]. Studies using transgenic animals overexpressing GAP-43 have identified GAP-43 as an intrinsic determinant of axonal regeneration $[11,12]$.

Cyclin-dependent kinase 5 (Cdk5) is serine/threonine protein kinase and its function in neural tissue is known to be involved in neuronal development, learning and memory, addictive behavior, and neurodegeneration [13-15]. At cellular level, $\mathrm{Cdk} 5$ plays a role in corticogenesis during neural development which was identified by growth promoting activity of Cdk 5 on the neuritis of cultured neurons [16]. Cdk5 has a broad range of substrates (e.g., neurotrophic factors such as TrkB, MAP1B axon guidance molecule, modulator molecules in actin and microtubule dynamics, and motor function-related protein such as NUDEL), all of which are related to regenerative processes of injured axons directly or indirectly $[17,18]$. More than a decade ago, we reported that the production of Cdk5 was induced in axons of the facial nerve after crush injury [19]. While this report suggests de novo synthesis of Cdk5 in response to nerve injury, the neuronal expression and downstream effects of Cdk5 activity after axonal injury have not been investigated yet.

Here we report the induction of Cdk5 production in DRG neurons given SNI and identification of STAT3 as downstream effector that mediates neurite outgrowth. Inhibition of Cdk5 activity in DRG neurons resulted in a retardation of axonal regeneration, implying Cdk5 activity as an intrinsic factor promoting axonal regeneration.

\section{MATERIALS AND METHODS}

\section{Animals and Sciatic Nerve Surgery}

Sprague-Dawley rats (7 weeks of age, male; Samtako Inc., Seoul, Korea) were used for all experiments. Animals were maintained for 1 week before the experiment in an animal room with regulated temperature of $24^{\circ} \mathrm{C}$ and a 12 hour-light $/ 12$ hour-dark cycle. Rats were anesthetized by intraperitoneally injecting ketamine (80 $\mathrm{mg} / \mathrm{kg}$ ) and xylazine $(5 \mathrm{mg} / \mathrm{kg})$. The sciatic nerve was exposed in the middle thigh and crushed by compressing with jewel's forceps (Solco, Seoul, Korea) for 30 seconds twice. All protocols involving the care of live animals and their postoperative care were approved by the Daejeon University Institutional Animal Use and Care Committee (approval number: DJUARB2019-029) and were in accordance with the Animal-Use Statement and Ethics Committee Approval Statement for Animal Experiments provided by Daejeon University (Daejeon, Korea).

\section{DRG Sensory Neuron Culture}

Primary neuron culture was essentially the same as described previously [20]. Briefly, the DRG at lumbar levels 4 and 5 were isolated from rats which had undergone preconditioning SNI for 3 days, and dissociated cells $\left(1.5 \times 10^{5}\right)$ were cultured on $12-\mathrm{mm}$ coverslips in 24-well plates. For the experiments blocking Cdk5 activity, roscovitine $(10 \mu \mathrm{M})$ or dimethyl sulfoxide (DMSO) vehicle was treated for 24 hours prior to cell harvest. The length of neurite was measured by using i-Solutions software program (Image \& Microscope Technology, Daejeon, Korea).

\section{Transfection of Primary DRG Neurons}

Plasmid constructs pCMV-dn-Cdk5-GFP, pCMV-Cdk5-GFP, pRc/CMV-STAT3-S727A, pRc/CMV-STAT3-Y705F, and pCMV-GFP were all obtained from Addgene (Watertown, MA, USA). DRG neurons were transfected with plasmid constructs by using lipofectamine 2000 (Invitrogen, Waltham, MA, USA) as described by the manufacturer. Briefly, 24 hours before transfection, neurons $\left(2 \times 10^{5}\right)$ in $500 \mu \mathrm{L}$ of culture medium (BME, Sigma-Aldrich Chemical Co., St. Louis, MO, USA) were plated on $12-\mathrm{mm}$ coverslips in 24-well plate. Plasmid DNA $(1 \mu \mathrm{g})$ was gently mixed with $50 \mu \mathrm{L}$ of BME and incubated for 5 minutes at room temperature. DNA and lipofectamine solutions were 
mixed and incubated for 20 minutes at room temperature. They were added to each well containing cells and mixed by rocking the plate. After incubation for 24 hours at $37^{\circ} \mathrm{C}$ in $\mathrm{CO}_{2}$ incubator, cells were fixed for immunofluorescence staining.

\section{Western Blot Analysis}

DRGs and sciatic nerves were prepared from rats 1-7 days after SNI. All the procedures of western blotting including the preparations of cell lysates and sodium dodecyl sulfate-polyacrylamide gel electrophoresis were carried out as described previously [20]. We used anti-Cdk5 (polyclonal, 1:1,000; Cell Signaling Technology, Danvers, MA, USA), anti-p35 (1:1,000; Santa Cruz Biotech., Dallas, TX, USA), anti-STAT3 (1:1,000; Cell Signaling Technology), anti-pSTAT3 (Y705) (1:1,000; Cell Signaling Technology), anti-pSTAT3 (S727) (1:1,000; Cell Signaling Technology), and anti- $\beta$-actin (1:50,000; Sigma-Aldrich Chemical Co.) primary antibodies, and anti-rabbit HRP (1:5,000; Cell Signaling Technology) and anti-mouse HRP (1:5,000; Cell Signaling Technology) secondary antibodies were used in this study. Quantification of protein bands in the X-ray film images was determined by using the i-Solution software.

\section{Immunofluorescence Staining}

DRG neurons in tissue sections and cultured cells were subjected to immunofluorescence staining as described in our previous study [20]. Primary antibodies used were polyclonal antibodies for Cdk5 (1:400; Santa Cruz Biotech.), p35 (1:1,000; Santa Cruz Biotech.), pSTAT3 (Y705) (1:100; Cell Signaling Technology), pSTAT3 (S7275; Cell Signaling Technology) (1:100), and NF-200 (1:1,000; Sigma-Aldrich Chemical Co.) and monoclonal antibody for NF-200 (1:400; Sigma-Aldrich Chemical Co.). Fuorescein-goat anti-mouse (1:400; Molecular Probes, Eugene, OR, USA) and rhodamine-goat anti-rabbit (1:400; Molecular Probes) secondary antibodies were used in this study.

\section{In Vivo Injection of Roscovitine Into DRG}

Roscovitine was injected into the DRG of rats as described in previous studies [21,22]. Briefly, the mediodorsal surface of the skin between lower thorax and lumbar area was excised and exposed $4-5 \mathrm{~cm}$ in a longitudinal direction. After cutting the muscle and vertebrae with a marginal excision of $0.5-\mathrm{cm}$ length at both ends, the DRG at lumbar 4 and 5 were carefully exposed. Roscovitine (4 nmole) or an equivalent volume of DMSO was injected into the DRG with a flow rate of $0.5 \mu \mathrm{L} / \mathrm{min}$ for $4 \mathrm{~min}$ - utes by using micropump (Harvard Instrument, Holliston, MA, USA). The sciatic nerve was given crush injury immediately after the injection of roscovitine and prepared 3 days later to analyze axonal regeneration in longitudinal nerve sections. Fluorescence images were captured and transferred to the Adobe Photoshop Program. For quantification of regenerating axons, the number of NF-200-stained axons intersecting with vertical lines at different lengths in the distal nerve was counted and the ratio of the number of axons relative to that at the location of $100 \mu \mathrm{m}$ proximal to the injury site was determined. The values from 5 to 7 nonconsecutive nerve sections per animal were averaged.

\section{Statistical Analysis}

The data were presented as standard error of mean and were compared among experimental groups by using Student unpaired t-test, 1-way analysis of variance with Bonferoni test for multiple comparisons (GraphPad Prism 7.00, San Diego, CA, USA), and 2-way analysis of variance with Sidak multiple comparison test. Statistically significant differences were reported as ${ }^{*} \mathrm{P}<0.05,{ }^{*} \mathrm{P}<0.01$ or ${ }^{* *} \mathrm{P}<0.001$.

\section{RESULTS}

To investigate whether SNI affects on the production of Cdk5 and activator proteins p35/25 in DRG neurons, we analyzed these proteins by western blot analysis. Moderate level of Cdk 5 was detected from untreated control animals and the levels were elevated after SNI (Fig. 1A). p35 was highly expressed in the intact DRG tissue, decreased 1 day after SNI, and gradually increased 3-7 days later (Fig. 1B). In contrast, p25, a cleavage product of p35, was not detected in the intact DRG and gradually increased after SNI. We further investigated the cellular distribution of Cd5 and 35 proteins in the DRG by immunofluorescence staining. Cdk5 protein signals were weakly observed in NF-200-stained DRG neurons in the control group and clearly increased after SNI, displaying a complete colocalization with NF-200-stained neurons (Fig. 2A). The number of Cdk5-positive cells was significantly increased in the DRG after SNI compared to the control group (Fig. 2B). p35 signals were observed in NF-200-stainiend DRG neurons and also in nonneuronal cells (marked arrows in Fig. 2C). Quantitative comparison showed no significant difference in the number of p35-labeled cells between the control and $\mathrm{SNI}$ groups though the mean values were higher in the injury group than the control group by $67 \%(\mathrm{P}=0.2)$ (Fig. 2D).

Previous studies showed that STAT3 is phosphorylated by 


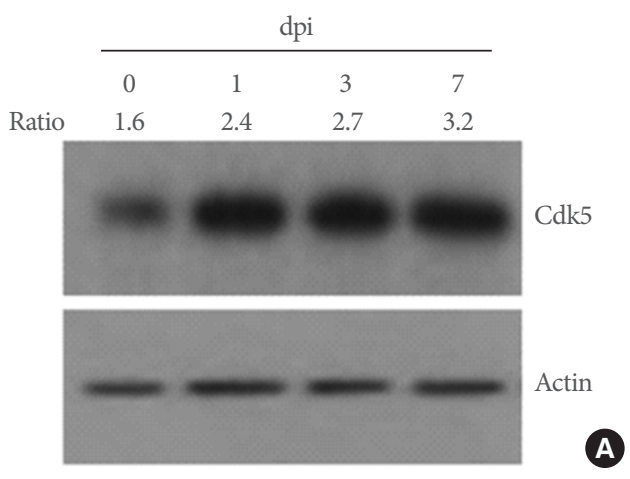

\begin{tabular}{ccccc} 
& \multicolumn{4}{c}{ dpi } \\
\cline { 2 - 5 } p35 Ratio & 0 & 1 & 3 & 7 \\
p25 Ratio & 0.01 & 0.2 & 0.4 & 0.8 \\
& 0.1 & 0.2 & 0.5
\end{tabular}

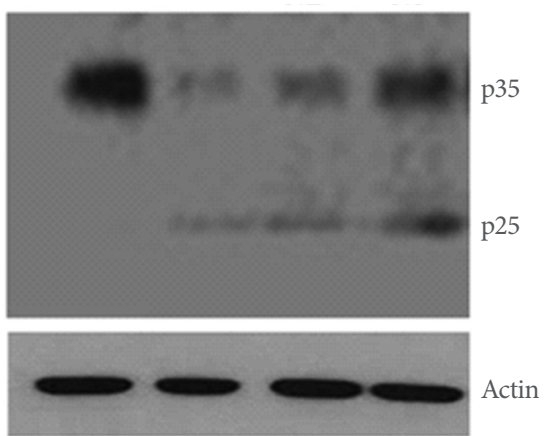

Fig. 1. Production of Cdk5 and p25 is elevated in dorsal root ganglion (DRG) tissues after sciatic nerve injury. DRG tissues prepared 0-7 day post injury (dpi) were used for western blot analysis for Cdk5 (A) and p35/25 (B) proteins. In panels A and B, western blotting for actin was performed as protein loading control. The numbers above western blot images denote the band intensity relative to actin.
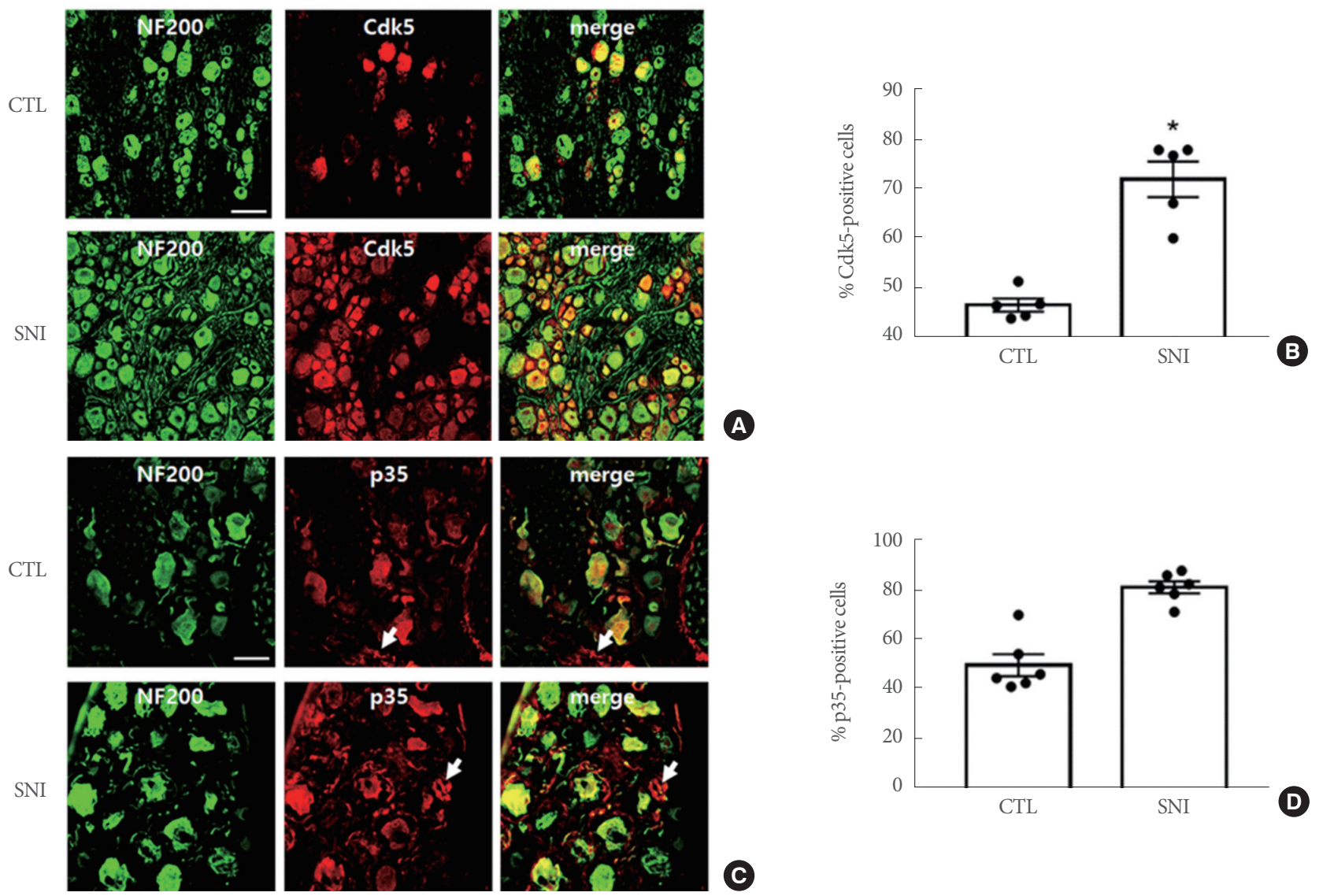

Fig. 2. Immunofluorescence view of Cdk5 and p35 protein signals in dorsal root ganglion (DRG) neurons. DRG tissues were prepared from untreated control animals (CTL) and animals given sciatic nerve injury (SNI) for 3 days. Representative images of immunofluorescence images for NF-200 and Cdk5 (A) and for NF-200 and p35 (C). Quantification of the number of neurons showing positive signals to Cdk5 and p35 in the control and SNI groups were plotted in panels B and D. ${ }^{\star} \mathrm{P}<0.05$ (error bar, standard error of the mean, $\mathrm{n}=5-6$ different replicates under 2 independent experiments). Scale bar represents $50 \mu \mathrm{m}$. 


\begin{tabular}{cccc} 
& \multicolumn{4}{c}{ dpi } \\
\cline { 2 - 4 } Ratio & 1 & 3 & 7 \\
2.3 & 2.4 & 2.1 & 1.9
\end{tabular}
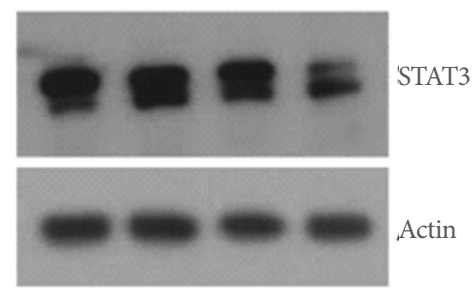
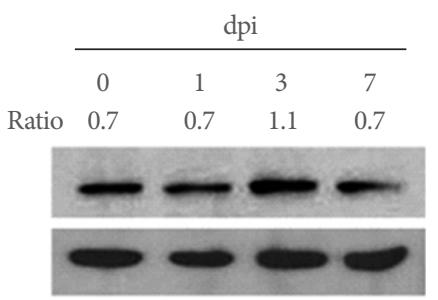

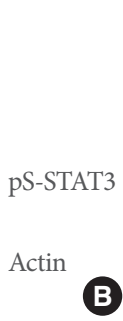

B
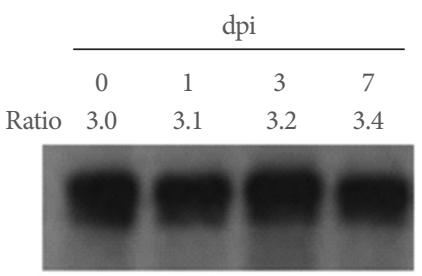

pY-STAT3

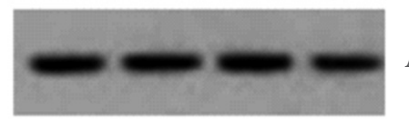

Actin

A

Fig. 3. Changes of STAT3 phosphorylation by sciatic nerve injury (SNI) in the dorsal root ganglion (DRG). DRG tissue lysates were prepared 0-7 days after SNI and used for western blotting for STAT3 (A), S727-p-STAT3 (pS-STAT3; B), and Y705-phospho-STAT3 (pY-STAT3; C). Western blotting for actin was performed as protein loading control. The numbers above western blot images denote the band intensity relative to actin.

CTL
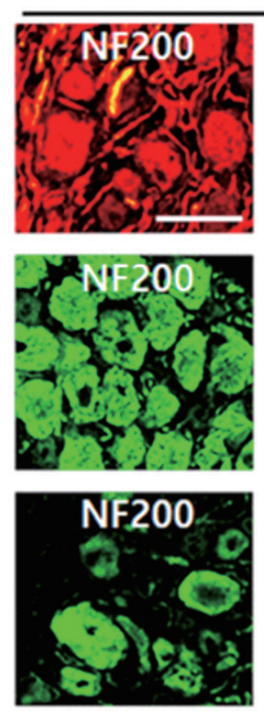
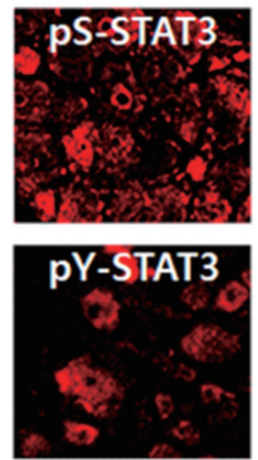

CTL
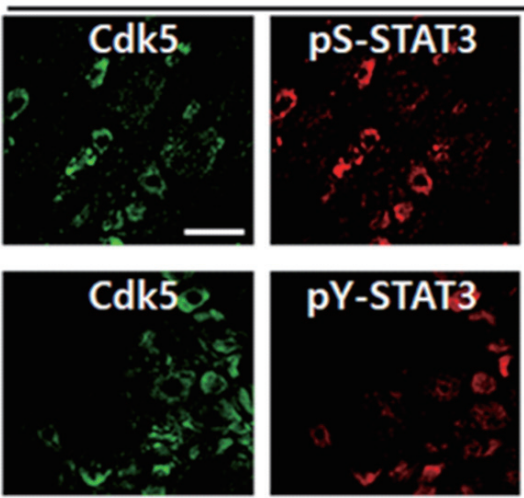
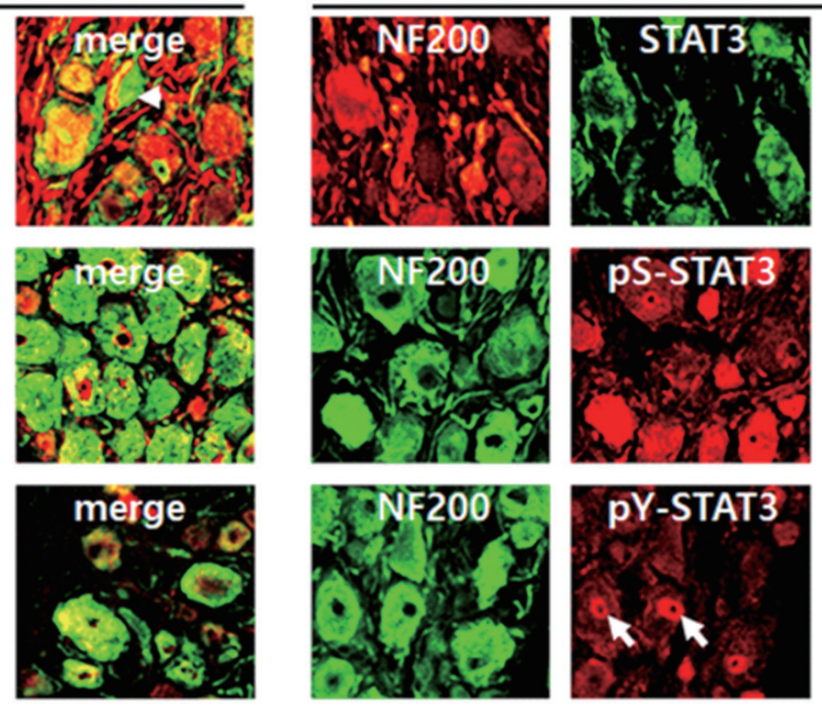

SNI
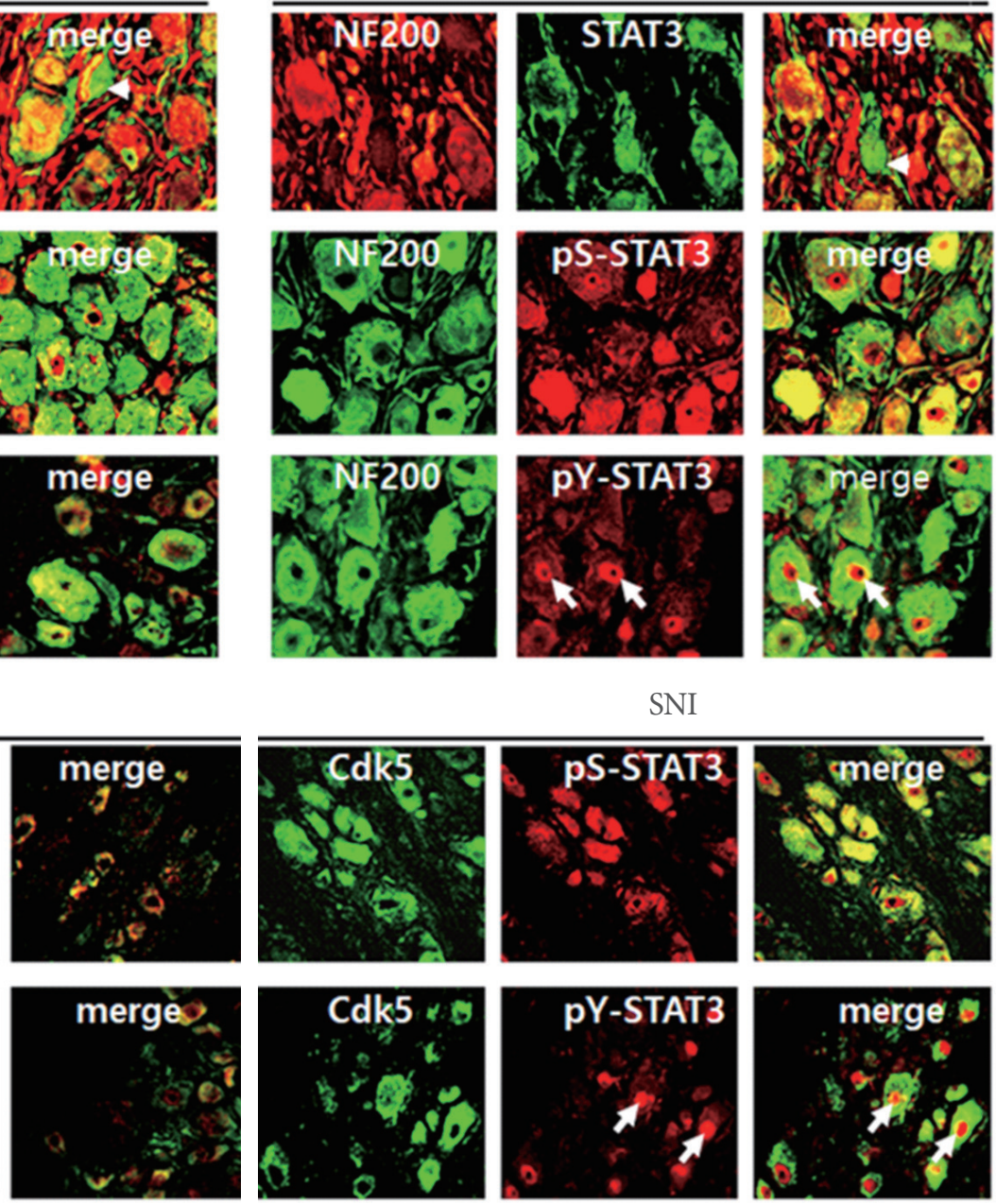

A

B

Fig. 4. Immunofluorescence view of STAT3, pS-STAT3, and pY-STAT3 signals in dorsal root ganglion (DRG) neurons. DRG tissues were prepared from untreated control animals (CTL) and animals given sciatic nerve injury (SNI) for 3 days. Representative images of immunofluorescence images for NF-200/STAT3, NF-200/pS-STAT3, and NF-200/pY-STAT3 in (A) and Cdk5/pS-STAT3 and Cdk5/ pY-STAT3 in (B). Scale bar represents $50 \mu \mathrm{m}$. 


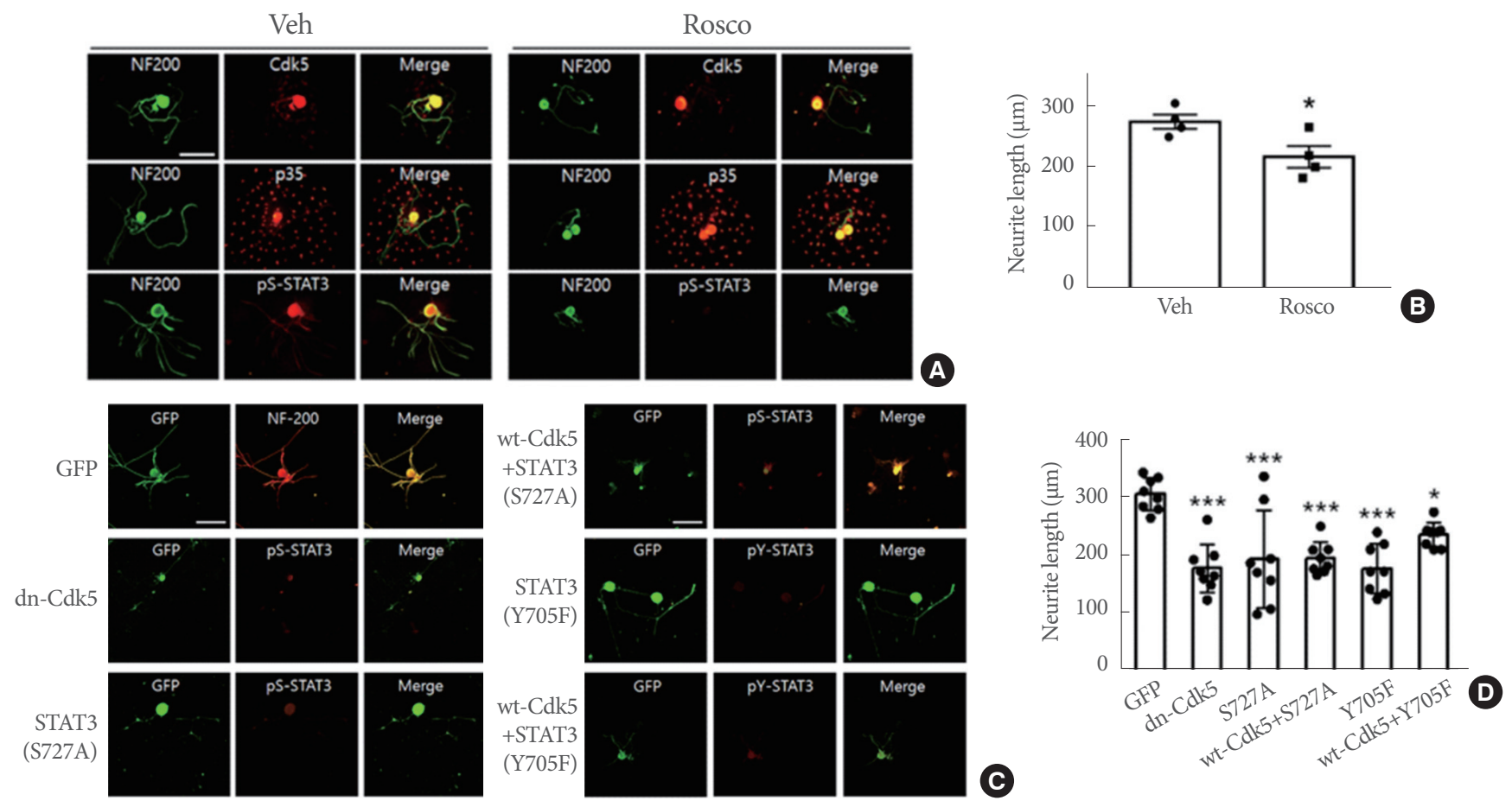

Fig. 5. Inhibition of Cdk5-STAT3 pathway in dorsal root ganglion (DRG) neurons attenuates neurite outgrowth. (A, B) Inhibition of STAT3 phosphorylation and neurite outgrowth in roscovitine-treated DRG neurons. Primary DRG neurons were treated with Cdk5 inhibitor roscovitine $(10 \mu \mathrm{M})$ or dimethyl sulfoxide (DMSO) vehicle for 24 hours prior to the cell harvest. Note that after roscovitine treatment, the intensity of pS-STAT3, but not Cdk5 and p35, signal was reduced. (B) A plot for quantitative comparison of neurite length in cell groups treated with roscovitine and DMSO vehicle. Neurite length was determined by analyzing randomly selected 10$15 \mathrm{DRG}$ neurons per experimental group. ${ }^{*} \mathrm{P}<0.05$ (Student t-test, $\mathrm{n}=4$ replicates in 2 independent experiments). (C, D) Inhibition of neurite outgrowth of DRG neurons after the transfection with the plasmids overexpressing dominant negative form of Cdk5 (dnCdk5), nonphosphorylatable STAT3 (S727A or Y705F), and wild-type Cdk5 (wt-Cdk5). Transfected cells were identified by GFP reporter. Representative immunofluorescence images for individual, transfected groups are shown in (C), and quantification of neurite outgrowth is plotted in (D). Mean neurite length was measured by analyzing 3-4 transfected cells and averaged for 8 replicates in 2 independent experiments $\left({ }^{*} \mathrm{P}<0.05,{ }^{* *} \mathrm{P}<0.01,{ }^{* *} \mathrm{P}<0.001,1\right.$-way analysis of variance). Scale bar represents $50 \mu \mathrm{m}$.

Cdk5 at serine 727 (S727) and after additional phosphorylation at tyrosine 705 (Y705), increases the transcription of target genes [23]. Here, we investigated whether Cdk5 activity is involved in STAT3 phosphorylation in DRG neurons. In the DRG, the basal level of STAT3 was high and remained unaltered after SNI (Fig. 3A). We also detected the basal level of phosphorylated STAT3 at S727 (hereafter pS-STAT3) in the DRG, which was increased 3 days after injury (Fig. 3B). In contrast, high level of phospho-STAT3 at Y705 (hereafter pYSTAT3) was observed in the control group (Fig. 3B) and the levels were not altered by SNI (Fig. 3C). When analyzed by immunofluorescence staining, STAT3 signals were mostly detected in NF-200-labeled DRG neurons, with some additional signals in nonneuronal cells in the control and SNI groups of animals (marked arrowheads in Fig. 4A). We found that pS-STAT3 signals, which were weakly observed in the control DRG neurons, were clearly increased after SNI. Finally, pY-STAT3 signals were detected in the DRG of both control and SNI groups of animals and found particularly in the nuclear zone of DRG neurons of SNI group of animals (marked arrows in Fig 4A). Both pS-STAT3 and pY-STAT3 signals were highly colocalized with Cdk5-labeled DRG neurons (Fig 4B). Here again, it was confirmed that in the DRG from SNI group of animals, most of pS-STAT3 and Cdk5 signals were evenly seen in neuronal cell bodies whereas pY-STAT3 signals were concentrated within the central nuclear zone area.

To further examine the effects of STAT3 phosphorylation on axonal elongation, we prepared primary DRG neurons. Here, both Cdk5 and p35 signals were clearly observed and were not affected by roscovitine treatment (Fig. 5A). p35 signals were 

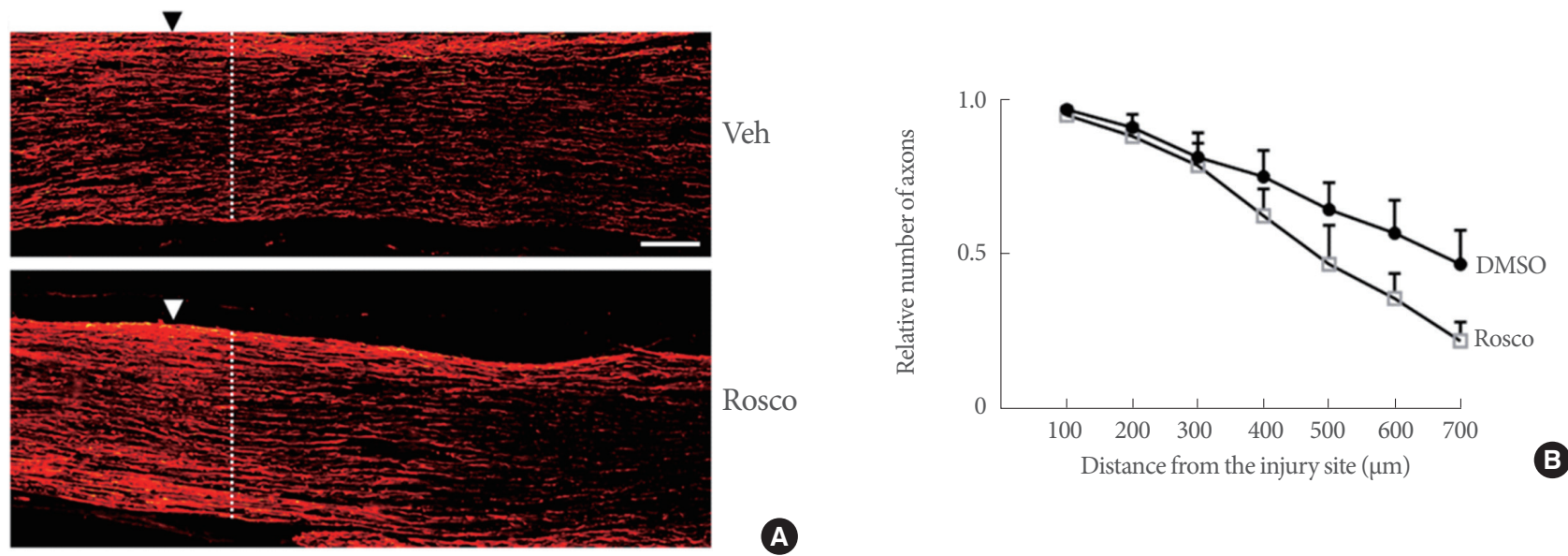

Fig. 6. Retardation of axonal regeneration by in vivo roscovitine treatment. Three days after the administration of roscovitine ( $4 \mathrm{nmoles})$ or dimethyl sulfoxide (DMSO) vehicle (Veh), longitudinal nerve sections were prepared for immunofluorescence analysis of regenerating axons. Representative immunofluorescence views of NF-200-stained axons for vehicle- and roscovitine (ROSCO)-injected groups are shown in (A), and in (B), the ratio of the number of regenerating axons in the distal nerve relative to that of axons in the proximal nerve was compared at different lengths between roscovitine (ROSCO)- and DMSO vehicle injected groups. $\mathrm{P}<0.001(\mathrm{~F}[6,28]=6.547,2$-way analysis of variance, $n=4$ animals per group). Vertical arrows in (A) denote the site of sciatic nerve injury. Scale bar represents $200 \mu \mathrm{m}$.

observed additionally in cells around the DRG neuronal cell body, possibly reflecting p35 expression in nonneuronal cells in the DRG such as Schwann cells and satellite cells. pS-STAT3 signals were clearly decreased by roscovitine treatment compared to the control, as the neurite outgrowth was significantly attenuated by roscovitine (Fig. 5B). We further investigated the effects of Cdk5 phosphorylation of STAT3 on neurite outgrowth in neurons transfected with dominant negative form of Cdk5 (dnCdk5) and nonphosphorylatable forms of STAT3. Cells transfected with dn-Cdk5 or STAT3 (S727A), as identified by GFP-fusion protein and cotransfected GFP, respectively revealed very weak $\mathrm{pS}$-STAT3 signals, indicating the inhibition of STAT3 phosphorylation at S727 (Fig. 5C). Similarly, STAT3 phosphorylation at $\mathrm{Y} 705$ were largely suppressed by overexpression of STAT3 (Y705F). Cell groups expressing dn-Cdk5, STAT3 (S727A), and STAT3 (Y705F) showed significant reductions in neurite growth compared to the control group that expresses GFP (Fig. 5D). In addition, cells transfected with the plasmid constructs of wild type Cdk5 along with STAT3 (S727A) showed similar levels of reduction in neurite outgrowth as those transfected with STAT3 (S727A). However, cotransfection with wt-Cdk5 and STAT3 (Y705F) was less effective at reducing neurite outgrowth than STAT3 (Y705F) transfection alone, showing a $33 \%$ of difference $(\mathrm{P}=0.14)$.

To examine whether Cdk5 activity in DRG neurons is required for axonal elongation in the sciatic nerve after injury, we injected roscovitine into the DRG to inhibit Cdk5 activity in vivo. As shown in Fig. 6A, the sciatic nerve treated with roscovitine showed a reduction of distal elongation of regenerating axons compared to DMSO-vesicle injected group. Differences in distal elongation of regenerating axons was clearly observed at $600 \mu \mathrm{m}$ or further distal portion of the nerve, showing a statistical significance between the groups $(\mathrm{P}<0.001)$ (Fig. 6B).

\section{DISCUSSION}

In this study, we demonstrate that Cdk5 activity is upregulated from DRG neurons in rats given SNI and phosphorylates STAT3. Since the lesion signal after PNI engenders cell body response including gene expression as the process of inducing axonal regeneration, our finding suggests that SNI-induced Cdk5-STAT3 signaling pathway may play a role in mediating the production of regeneration-inducing factors at gene expression level.

An injury on the peripheral nerve induces local pathological responses in nonneuronal cells such as Schwann cells and macrophages as well as injured axons, and combined lesion signal is transmitted into the neuronal cell body [3]. Since we found increases in Cdk5 protein level in DRG sensory neurons, the lesion signal may induce Cdk5 gene expression at transcriptional and/or posttranscriptional levels. Interestingly however, high level of p35, an activator protein of Cdk5, was observed in the DRG of untreated control animals. Cdk5 is expressed in several 
brain regions and its activity is regulated by p35/p25 that is induced by external stimulation $[13,24,25]$. Thus, the expression of Cdk5 and p35 in the peripheral nerve seems to be regulated in a different manner from the brain. It was also noted that p25, a cleavage product of $\mathrm{p} 35$ by calpain, was not observed in the control DRG and induced after nerve injury. Although p25, like p35, binds to and activates Cdk5, it is localized in the cytosol and induces Cdk5 phosphorylation of substrate proteins such as Tau and neurofilament $\mathrm{H}$ that are related to neurotoxic effect in degenerating neurons including Alzheimer disease brain, which is thus distinct in substrate specificity from p35-Cdk5 activity [26]. More recently, it was reported that Cdk5/p25 increased beta site amyloid precursor protein cleaving enzyme leading to amyloidogenesis in relation to Alzheimer disease [27]. Our data strongly suggest that both Cdk5 and p25 are increased after PNI and play a role in axonal regeneration. Given that one of the main functions of Cdk5-p25 is to mediate apoptosis particularly in neurons undergoing degeneration in Alzheimer, Parkinson, and amyotrophic lateral sclerosis brain [14,28,29], it remains to be determined how Cdk5/p25 activity, in contrast to its neurotoxicity effects in the brain, mediates regenerative response.

Our data showed that the Cdk5 activity is increased in DRG sensory neurons after SNI and phosphorylates STAT3 at serine 727. Cdk5 phosphorylation of STAT3 at serine 727 has been shown in neural tissues and cancer cells $[23,30]$, and here we present Cdk5 activity phosphorylating STAT3 in DRG neurons. Recently, we found that Cdk5 phosphorylation of STAT3 in regenerating axons after SNI is related to enhanced mitochondrial activity in axons (Hwang and Namgung, unpublished data). We speculate that phospho-STAT3 may play a role in mediating axonal regeneration possibly by acting as a transcription factor, as shown in regenerating axons of retinal ganglion cells [31]. Given that STAT3 phosphorylation at Y705 is required for the activation of STAT3, we measured pY-STAT3 in DRG neurons and found its constitutive expression in the DRG before and after SNI. Yet, the immunofluorescence localization of pY-STAT3 revealed its concentric localization in the nucleus of DRG neurons after SNI. It was previously shown that STAT3 activation as a transcription factor requires the phosphorylation at serine 727 residue for its maximal activity in addition to Y705 [32], raising the possibility that the injury-induced increases in phosphorylation of STAT3 at S727 may facilitate the translocation of pY-STAT3 into the nucleus leading to transcriptional activation for target gene expression. This speculation of STAT3 function is supported by our data demonstrating that the pharmacologi- cal and genetic blockades of Cdk5 activity resulted in retarded neurite outgrowth in cultured DRG neurons. Inhibition of Cdk5 activity or STAT3 phosphorylation at S727 and Y705 residues resulted in reduced neurite outgrowth, suggesting that Cdk5-STAT3 pathway may be involved in neurite outgrowth.

We found that axonal regeneration after SNI was significantly decreased by in vivo administration of roscovitine into the DRG. While our data suggest that roscovitine may inhibit Cdk5 activity in DRG neurons in vivo, a possibility of roscovitine inhibition of other Cdk family proteins such as Cdc2 cannot be excluded. Cdc2 expression is strongly induced from Schwann cells in the sciatic nerve after injury and was shown to be involved in axonal regeneration [33]. Whether or not Cdc2 expression is induced in the DRG after PNI and its activity is linked to regenerative responses of the peripheral branch of the injured nerve remains to be determined.

In conclusion, we demonstrate that $\mathrm{Cdk} 5$ activity induced from DRG neurons after SNI increased STAT3 activity. STAT3 activity in DRG neurons may play a role in promoting axonal regeneration after PNI and thus implicates therapeutic application as an intrinsic factor of peripheral nerve repair.

\section{AUTHOR CONTRIBUTION STATEMENT}

- Conceptualization: JH, UN

- Formal Analysis: JH

- Investigation: $\mathrm{JH}$

- Methodology: $J H$

- Project Administration: UN

- Writing - Original Draft: $U N$

- Writing - Review \& Editing: $U N$

\section{REFERENCES}

1. Silver J, Schwab ME, Popovich PG. Central nervous system regenerative failure: role of oligodendrocytes, astrocytes, and microglia. Cold Spring Harb Perspect Biol 2014;7:a020602.

2. He Z, Jin Y. Intrinsic control of axon regeneration. Neuron 2016;90: 437-51.

3. Chen ZL, Yu WM, Strickland S. Peripheral regeneration. Annu Rev Neurosci 2007;30:209-33.

4. Mahar M, Cavalli V. Intrinsic mechanisms of neuronal axon regeneration. Nat Rev Neurosci 2018;19:323-37.

5. Namgung U. The role of Schwann cell-axon interaction in peripheral nerve regeneration. Cells Tissues Organs 2014;200:6-12. 
6. Jessen KR, Mirsky R, Lloyd AC. Schwann cells: development and role in nerve repair. Cold Spring Harb Perspect Biol 2015;7:a020487.

7. Lee JK, Geoffroy CG, Chan AF, Tolentino KE, Crawford MJ, Leal $\mathrm{MA}$, et al. Assessing spinal axon regeneration and sprouting in Nogo-, MAG-, and OMgp-deficient mice. Neuron 2010;66:663-70.

8. Meyer M, Matsuoka I, Wetmore C, Olson L, Thoenen H. Enhanced synthesis of brain-derived neurotrophic factor in the lesioned peripheral nerve: different mechanisms are responsible for the regulation of BDNF and NGF mRNA J Cell Biol. 1992;119:4554.

9. García-Villamar V, Hernández-Aragón LG, Chávez-Ríos JR, Ortega A, Martínez-Gómez M, Castelán F. Expression of glial cell linederived neurotrophic factor (GDNF) and the GDNF family receptor alpha subunit 1 in the paravaginal ganglia of nulliparous and primiparous rabbits. Int Neurourol J 2018;22:S23-33.

10. Skene JH. Axonal growth-associated proteins. Annu Rev Neurosci 1989;12:127-56.

11. Aigner L, Arber S, Kapfhammer JP, Laux T, Schneider C, Botteri F, et al. Overexpression of the neural growth-associated protein GAP43 induces nerve sprouting in the adult nervous system of transgenic mice. Cell 1995;83:269-78.

12. Bomze HM, Bulsara KR, Iskandar BJ, Caroni P, Skene JH. Spinal axon regeneration evoked by replacing two growth cone proteins in adult neurons. Nat Neurosci 2001;4:38-43.

13. Lew J, Huang QQ, Qi Z, Winkfein RJ, Aebersold R, Hunt T, et al. A brain-specific activator of cyclin-dependent kinase 5. Nature 1994; 371:423-6.

14. Dhavan R, Tsai LH. A decade of CDK5. Nat Rev Mol Cell Biol 2001;2:749-59.

15. Yang SH. Cellular and molecular mediators of neuroinflammation in Alzheimer disease. Int Neurourol J 2019;23:S54-62.

16. Nikolic M, Dudek H, Kwon YT, Ramos YF, Tsai LH. The cdk5/p35 kinase is essential for neurite outgrowth during neuronal differentiation. Genes Dev 1996;10:816-25.

17. Ye T, Fu AK, Ip NY. Cyclin-dependent kinase 5 in axon growth and regeneration. Int Rev Neurobiol 2012;105:91-115.

18. Hur EM, Lee BD. Microtubule-targeting agents enter the central nervous system (CNS): double-edged swords for treating CNS injury and disease. Int Neurourol J 2014;18:171-8.

19. Namgung U, Choi BH, Park S, Lee JU, Seo HS, Suh BC, et al. Activation of cyclin-dependent kinase 5 is involved in axonal regeneration. Mol Cell Neurosci 2004;25:422-32.

20. Chang IA, Oh MJ, Kim MH, Park SK, Kim BG, Namgung U. Vimentin phosphorylation by Cdc2 in Schwann cell controls axon growth via $\beta 1$-integrin activation. FASEB J 2012;26:2401-13.
21. Cheah M, Fawcett JW, Andrews MR. Dorsal root ganglion injection and dorsal root crush injury as a model for sensory axon regeneration. J Vis Exp 2017;123:55535.

22. Samad OA, Tan AM, Cheng X, Foster E, Dib-Hajj SD, Waxman SG. Virus-mediated shRNA knockdown of $\mathrm{Na}(\mathrm{v}) 1.3$ in rat dorsal root ganglion attenuates nerve injury-induced neuropathic pain. Mol Ther 2013;21:49-56.

23. Fu AK, Fu WY, Ng AK, Chien WW, Ng YP, Wang JH, et al. Cyclindependent kinase 5 phosphorylates signal transducer and activator of transcription 3 and regulates its transcriptional activity. Proc Natl Acad Sci U S A 2004;101:6728-33.

24. Tsai LH, Delalle I, Caviness VS Jr, Chae T, Harlow E. p35 is a neural-specific regulatory subunit of cyclin-dependent kinase 5 . Nature 1994;371:419-23.

25. Wu DC, Yu YP, Lee NT, Yu AC, Wang JH, Han YF. The expression of Cdk5, p35, p39, and Cdk5 kinase activity in developing, adult, and aged rat brains. Neurochem Res 2000;25:923-9.

26. Patrick GN, Zukerberg L, Nikolic M, de la Monte S, Dikkes P, Tsai LH. Conversion of p35 to p25 deregulates Cdk5 activity and promotes neurodegeneration. Nature 1999;402:615-22.

27. Wen Y, Yu WH, Maloney B, Bailey J, Ma J, Marié I, et al. Transcriptional regulation of beta-secretase by $\mathrm{p} 25 / \mathrm{cdk} 5$ leads to enhanced amyloidogenic processing. Neuron 2008;57:680-90.

28. Camins A, Verdaguer E, Folch J, Canudas AM, Pallàs M. The role of CDK5/P25 formation/inhibition in neurodegeneration. Drug News Perspect 2006;19:453-60.

29. Alvira D, Ferrer I, Gutierrez-Cuesta J, Garcia-Castro B, Pallàs M, Camins A. Activation of the calpain/cdk5/p25 pathway in the girus cinguli in Parkinson's disease. Parkinsonism Relat Disord 2008;14:309-13.

30. Courapied S, Sellier H, de Carné Trécesson S, Vigneron A, Bernard AC, Gamelin E, et al. The cdk5 kinase regulates the STAT3 transcription factor to prevent DNA damage upon topoisomerase I inhibition. J Biol Chem 2010;285:26765-78.

31. Luo X, Ribeiro M, Bray ER, Lee DH, Yungher BJ, Mehta ST, et al. Enhanced transcriptional activity and mitochondrial localization of STAT3 co-induce axon regrowth in the adult central nervous system. Cell Rep 2016;15:398-410.

32. Wen Z, Zhong Z, Darnell JE Jr. Maximal activation of transcription by Stat 1 and Stat3 requires both tyrosine and serine phosphorylation. Cell 1995;82:241-50.

33. Han IS, Seo TB, Kim KH, Yoon JH, Yoon SJ, Namgung U. Cdc2mediated Schwann cell migration during peripheral nerve regeneration. J Cell Sci 2007;120:246-55. 